\title{
Manifestations of Varying Grading Level in CdSe/ZnSe Core Shell Nanocrystals
}

Kiran G. Sonawane, ${ }^{1,2}$ Kaustubh S. Agarwal, ${ }^{1}$ Chinmay Phadnis, ${ }^{1}$ Dharmendar Sharma, ${ }^{3}$ Arunasish Layek, ${ }^{3}$ Arindam Chowdhury, ${ }^{3,+}$ and Shailaja Mahamuni ${ }^{1, *}$

\section{Supporting Information}

\section{Experimental}

PL imaging and spectroscopy has been performed on a home built epi-fluorescence setup, details of which can be found elsewhere. ${ }^{1,2}$ In summary, a set up based on Nikon TE2000U inverted optical microscope with high numerical aperture oil immersion objective (Nikon Plan Apo 60x/ 1.49 NA, Oil) was used for PL imaging. The NCs were excited with $475 \mathrm{~nm}$ laser $\left(0.5-1 \mathrm{~kW} \mathrm{~cm}^{-2}\right)$ and the fluorescence emission is collected using an interline CCD camera (DVC-1412). Typically, $15 \times 15 \mu \mathrm{m}^{2}$ field of view was imaged, which contained several tens of individual nanocrystals (NCs) (FWHM $200 \mathrm{~nm}$ for emission spots).

\section{Computational}

Calculations based on Finite Element Method (FEM) ${ }^{3}$ were carried out to examine the variation in oscillator strength, and wavefunction on change in the shape of confinement potential. Schrödinger equation was solved for a spherically symmetric potential which is defined as. ${ }^{4}$

$$
V(r)=\left\{\begin{array}{cl}
V_{0} \frac{r^{v}}{r^{v}+\mathrm{b}^{v}} & \text { for } 0 \leq r \leq r_{s} \\
\infty & \text { for } r>r_{s}
\end{array}\right.
$$

Where $\mathrm{r}$ is the radial coordinate, ' $v$ ' determines the steepness of the potential, and ' $b$ ' is FWHM of the potential. We have used ' $b$ ' as the approximate core radius $\left(r_{\mathrm{c}}\right)$ of the quantum dot 
(QD) due to the grading present in CdSe-ZnSe, $r_{\mathrm{s}}$ is the shell boundary and $\mathrm{V}_{0}$ is the value of the potential when $r=r_{\mathrm{s}} . \mathrm{V}_{0}$ is estimated from the band gap offset ratio between CdSe-ZnSe and bulk band gap of CdSe. The offset ratio between CdSe-ZnSe is considered as A:B $=11: 1$ and $\mathrm{A}+\mathrm{B}=1$. Thus, $\mathrm{V}_{0}$ for electron is $\mathrm{A} * \mathrm{E}_{\mathrm{g}}$ and $\mathrm{Vo}$ for hole is $\mathrm{B} * \mathrm{E}_{\mathrm{g}}^{5}$ where $\mathrm{E}_{\mathrm{g}}$ is the bulk band gap of CdSe $(1.74 \mathrm{eV})$.

The smallest value of $v$ corresponds to a slowly varying potential whereas increasing $v$ would clearly tend potential to vary rapidly. Four different shapes of confinement potentials are used. $v=3$ is the most slowly varying confinement potential and similar to Graded- $48 . v=10$ is also slowly varying but with a lesser degree, and represents Graded-36. A flat confinement potential represents alloy structure. The calculations are also performed on abrupt core/shell sample with CdSe at core and $\mathrm{ZnSe}$ at shell.

Since the potential is spherically symmetric, the radial Schrödinger equation is,

$$
\frac{1}{r^{2}} \frac{d}{d r}\left(r^{2} \frac{d \Psi(r)}{d r}\right)-\frac{2 m^{*}}{\hbar^{2}}[V(r)-E] \Psi(r)=l(l+1) \Psi(r)
$$

Where $\Psi(r)$ denotes the radial part of orbital wave function

Since we are interested in $\mathrm{n}-\mathrm{S}$ states, we have considered $l=0$. In order to incorporate FEM, the above equation was brought into the dimensionless form by scaling the radial coordinate $r$ as $\mathrm{nm}$ and energy $\mathrm{E}$ as $\mathrm{eV}$. The values for the parameters are as follows. The radius of the quantum dot is $r_{s}=2.45 \mathrm{~nm}$, for electron $\mathrm{m}^{*}=0.13 \mathrm{~m}_{\mathrm{e}}$ and for hole $\mathrm{m}^{*}=0.44 \mathrm{~m}_{\mathrm{e}}$, and ' $\mathrm{v}^{\prime}$ is varied as 3 and 10 . The boundary condition for the envelope wave function $\Psi(r)$ is that $\Psi(r)$ $=0$ when $r=r_{\mathrm{s}}$.

An OCTAVE code was employed to compute the wave function using FEM. Linear interpolation was used to interpolate the wave functions since the size of each element in our domain $(0<r<2.45 \mathrm{~nm})$ is small. 


\section{Single particle PL imaging and spectroscopy}

\section{Sample Preparation:}

For single NC photoluminescence imaging and spectroscopy measurements, very dilute ( $\sim \mathrm{nM})$ suspension of $\mathrm{NCs}$ in $\mathrm{CHCl}_{3}$ and polymethylmethacrylate (PMMA) matrix was spin cast at $\sim 2000 \mathrm{rpm}$ onto a freshly cleaned microscope coverslips (Corning, $22 \times 22$ No.1). The density of NCs was optimized by varying concentration $(0.4-1.0 \mathrm{nM})$ to obtain well separated (more than $1 \mu \mathrm{m})$ and randomly distributed diffraction limited emission spots of NCs.

\section{PL imaging}

Fluorescence intermittency (blinking) of spatially well separated $(>1 \mu \mathrm{m})$ diffractionlimited spots was probed by collecting movies (16 bit image stacks) at $20 \mathrm{~Hz}(50 \mathrm{~ms}$ CCD exposure) for 2-10 min.

\section{PL Spectroscopy}

For spectral measurements of individual NCs, combination of slit and transmission grating was used between objective and CCD. We chose a low groove density $(70 \mathrm{l} / \mathrm{mm}$, Optometrics) TG which transmits $\sim 40 \%$ of light in $0^{\text {th }}$ order and $32 \%$ light in $1^{\text {st }}$ order, having the added benefit of minimal light loss. The diffracted light from TG is then detected by CCD camera mounted on a $x-y-z$ adjustable stage, and the distance between the TG and the CCD is maintained such that both the non-dispersed image $\left(0^{\text {th }}\right.$ order diffraction) and the dispersed spectra $\left(1^{\text {st }}\right.$ order diffraction) can be detected simultaneously using the same CCD chip $\left(1412 \times 1040\right.$ pixels, $9.1 \times 6.7 \mathrm{~mm}^{2}$ in dimension). Slit width was selected such that only 5-6 individual spots $\left(\right.$ zero $^{\text {th }}$ order) and their corresponding horizontal streaks (spectra) could be obtained in one shot. High exposure time (500 to $2000 \mathrm{~ms}$ ) was used to collect spectra, and data were obtained in digital format. 


\section{Data Analyses}

Analysis of data in digital video and image format was carried out using freely available ImageJ (NIH) and Origin8. PL images were background flattened using a rolling ball algorithm due to slight modulations in the excitation field. The intensity time traces for blinking analysis were obtained for over an area $(3 \times 3$ pixel) surrounding the emission spot using plotting ' $z$ profile' in ImageJ and data extracted in ASCII format for further analysis. Only well separated diffraction limited spots were analyzed to ensure that emission profile is generated from single emitter which shows single step photo blinking and photo bleaching. The spectral profiles from spectrally resolved images were obtained by integrating 3 pixels along vertical directions and an average background of the same dimensions was subtracted to obtain the emission profile. Pixel to $\mathrm{nm}$ calibration is carried out using three laser lines: $488 \mathrm{~nm}, 532 \mathrm{~nm}$ and $633 \mathrm{~nm}$. For this setup minimum energetic resolution obtained was $\sim 5 \mathrm{~nm}$, which corresponds to $\sim 20 \mathrm{meV}$ for the given wavelength range. The spectra were further corrected for CCD response over the entire wavelength range $(450-750 \mathrm{~nm})$. The spectral information such as peak positions and FWHM for each spectrum was obtained by fitting a polynomial function in Origin.

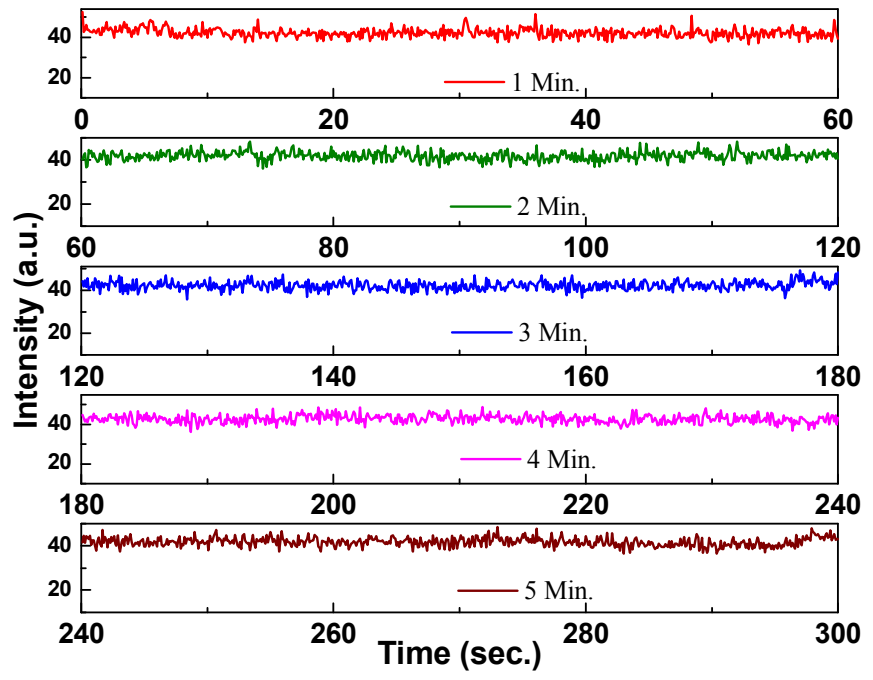


Figure SI. Blinking trace of Graded-48 recorded with 50ms integration time.

\section{Long time blinking trace small integration time:}

Figure SI represents data collected on Graded-48 NCs for $5 \mathrm{~min}$. The integration time for the data is $50 \mathrm{~ms}$. It is clear from the figure that even with such a small integration time there is no off state. Fast blinking in these NCs is not present.

\section{TEM Measurement:}
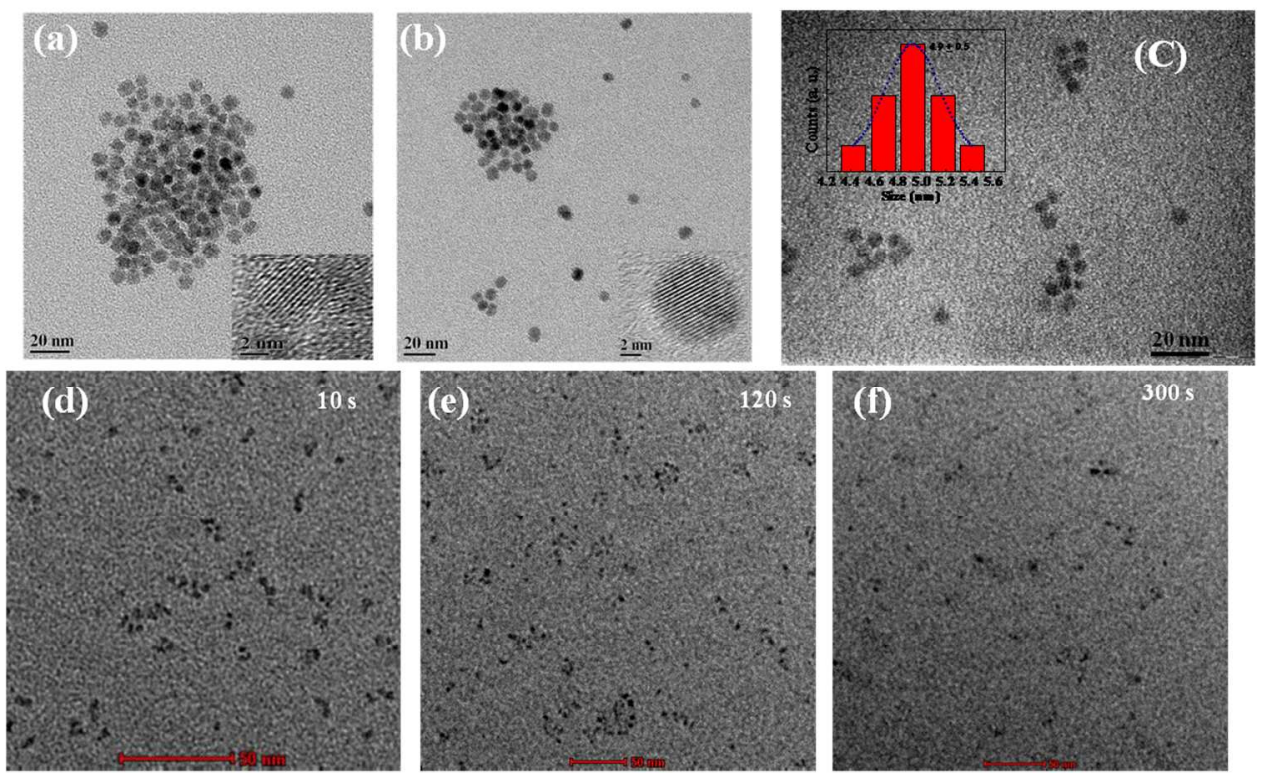

FIG. SII TEM images (a) Graded-36, size $4.5 \pm 0.5 \mathrm{~nm}$ (b)Alloy-36, size $4.9 \pm 0.8 \mathrm{~nm}$ (c) Histogram TEM and Histogram for Graded-48, size 4.9 \pm 0.5 (d) Graded-36, after 10 s reaction time, size $2.3 \pm 0.8$ (e) Graded-36, after $120 \mathrm{~s}$ reaction time, size $3.3 \pm 0.5$ (f) Graded-36 after $300 \mathrm{~s}$ reaction time, size $3.6 \pm 0.5$.

Spectra of ensemble and spectra produced by adding all emission spectra of individual NCs: 
Figure SIII represent normalized ensemble spectra which are obtained by adding spectra of individual NCs. It is worthwhile to note that the ensemble and addition of individual spectrum matches closely. These findings indicate that NCs contributing to different spectral region of ensemble has been taken care of.

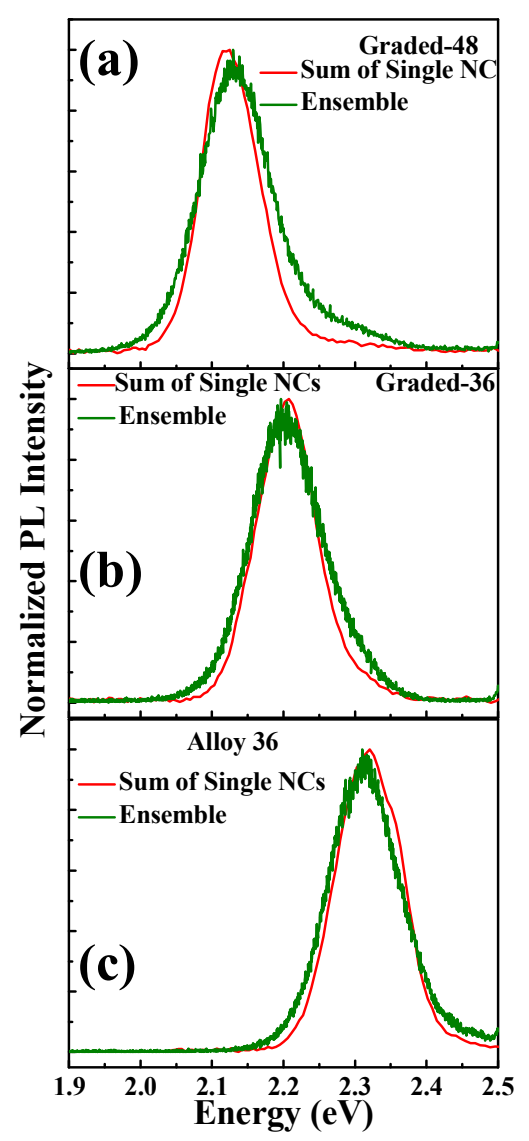

FIG. SIII Sum of individual NC spectrum plotted with ensemble emission spectrum for (a)Graded-48, (b) Graded-36, (c) Alloy-36.

\section{Blinking movies:}

Movies M1, M2, and M3 represent blinking movies for Graded-48, Graded-36 and Alloy-36, respectively. 


\section{References:}

1. Layek, A.; De, S.; Thorat, R.; Chowdhury, A. Spectrally Resolved Photoluminescence Imaging of ZnO Nanocrystals at Single-Particle Levels. J. Phys. Chem. Lett. 2011, 2, 1241-1247.

2. De, S.; Layek, A.; Raja, A.; Kadir, A.; Gokhale, M.; Bhattacharya, A.; Dhar, S.; Chowdhury, A. Two Distinct Origins of Highly Localized Luminescent Centers within InGaN/GaN Quantum-Well Light-Emitting Diodes. Adv. Funct. Mater. 2011, 21, 38283835.

3. Ram-Mohan, R. Finite Element and Boundary Element Applications to Quantum Mechanics; Oxford University Press: NY, U.S.A., 2002.

4. Cragg, G. E.; Efros, A. L. Suppression of Auger Processes in Confined Structures. Nano Lett. 2010, 10, 313-317.

5. Mukherjee, A.; Ghosh, S. Optimum Excitation Photon Energy For Cdse-Zns Core-Shell Quantum Dot Based Luminescence Imaging. J. Phys. D: Appl. Phys. 2012, 45, 195103.

6. Smith, A. M.; Lane, L. A.; Nie, S. Mapping The Spatial Distribution Of Charge Carriers In Quantum-Confined Heterostructures. Nature Commun. 2014, 5, 4506. 